\title{
On the energy growth of some periodically driven quantum systems with shrinking gaps in the spectrum
}

\author{
Pierre Duclos ${ }^{1}$, Ondra Lev ${ }^{2}$, Pavel Štovíček ${ }^{2}$
}

October 29, 2018

\begin{abstract}
${ }^{1}$ Centre de Physique Théorique de Marseille UMR 6207 - Unité Mixte de Recherche du CNRS et des Universités Aix-Marseille I, Aix-Marseille II et de l' Université du Sud Toulon-Var - Laboratoire affilié à la FRUMAM

${ }^{2}$ Department of Mathematics, Faculty of Nuclear Science, Czech Technical University, Trojanova 13, 12000 Prague, Czech Republic
\end{abstract}

\begin{abstract}
We consider quantum Hamiltonians of the form $H(t)=H+V(t)$ where the spectrum of $H$ is semibounded and discrete, and the eigenvalues behave as $E_{n} \sim n^{\alpha}$, with $0<\alpha<1$. In particular, the gaps between successive eigenvalues decay as $n^{\alpha-1}$. $V(t)$ is supposed to be periodic, bounded, continuously differentiable in the strong sense and such that the matrix entries with respect to the spectral decomposition of $H$ obey the estimate $\left\|V(t)_{m, n}\right\| \leq \varepsilon|m-n|^{-p} \max \{m, n\}^{-2 \gamma}$ for $m \neq n$ where $\varepsilon>0$, $p \geq 1$ and $\gamma=(1-\alpha) / 2$. We show that the energy diffusion exponent can be arbitrarily small provided $p$ is sufficiently large and $\varepsilon$ is small enough. More precisely, for any initial condition $\Psi \in \operatorname{Dom}\left(H^{1 / 2}\right)$, the diffusion of energy is bounded from above as $\langle H\rangle_{\Psi}(t)=O\left(t^{\sigma}\right)$ where $\sigma=\alpha /\left(2\lceil p-1\rceil \gamma-\frac{1}{2}\right)$. As an application we consider the Hamiltonian $H(t)=|p|^{\alpha}+\varepsilon v(\theta, t)$ on $L^{2}\left(S^{1}, \mathrm{~d} \theta\right)$ which was discussed earlier in the literature by Howland.
\end{abstract}

\section{Introduction}

One of the basic questions one can ask about time-dependent quantum systems is the growth of energy on a long time scale for a given initial condition. Unfortunately the quantum dynamics in the time-dependent case proved itself to be rather difficult to analyze in its full generality and complexity. The systems which allow for at least partially analytical treatment and whose dynamics has been perhaps best studied from various points of view are 
either driven harmonic oscillators [4, 17, 10, 15] or periodically kicked quantum Hamiltonians [11, 12, 5, 7, 8, 25]. On a more general level, it is widely believed that there exist close links between long time behavior of a quantum system and its spectral properties. For time-independent quantum systems such a relation is manifested by the famous RAGE theorem, see [30] for a summary and references to the original papers. In a modified form this theorem has been extended to periodic and quasi-periodic quantum systems [15, 21, 28]. In this case the relevant operator whose spectral properties are of interest is the Floquet (monodromy) operator. Naturally, much attention has been paid to the spectral analysis of Floquet operators in some of the papers cited above, see also [2] for more recent results. Let us mention that a refined analysis of how the spectral properties determine the quantum dynamics is now available, see for example [16, 9] and other papers, but here we are not directly concerned with this question.

Thus for periodically time-dependent systems one can distinguish as a related problem the spectral analysis of the Floquet operator under certain assumptions on the quantum Hamiltonian. Frequently one writes the time-dependent Hamiltonian in the form $H(t)=$ $H+V(t)$ while imposing assumptions on the spectral properties of the unperturbed part $H$ and requiring some sort of regularity from the perturbation $V(t)$. For our purposes an approach is rather important which is based on the adiabatic methods and which was initiated by Howland [18, 19] and further extended in [26, 22]. An essential property imposed on the unperturbed Hamiltonian in this case is the discreteness of the spectrum with increasing gaps between successive eigenvalues.

Under this hypothesis Nenciu in [27] was not only able to strengthen the results due to Howland but he derived in addition an upper bound on the diffusive growth of the energy having the form const $t^{a / n}$ where $a>0$ is given by the spectral properties of $H$ and $n$ is the order of differentiability of $V(t)$. Inspired by this result on the energy growth, Joye in [23] considered another class of time-dependent quantum Hamiltonians with rather mild assumptions on the spectral properties of $H$ but, on the other hand, assuming that the strength of the perturbation $V(t)$ is in some sense small with respect to $H$. Moreover, as far as the energy diffusion is discussed, the periodicity of $V(t)$ is required neither in [27] nor in [23].

It is worthwhile to mention that Howland in [20] succeeded to treat also the case when the spectrum of $H$ is discrete but the gaps between successive eigenvalues are decreasing. To achieve this goal he restricted himself to certain classes of perturbations $V(t)$ characterized by the behavior of matrix entries with respect to the eigen-basis of $H$. In particular, he discussed as an example the following model: $H(t)=|p|^{\alpha}+v(\theta, t)$ in $L^{2}\left(S^{1}, \mathrm{~d} \theta\right)$ where $0<\alpha<1$ and $v(\theta, t)$ is in $C^{\infty}\left(S^{1} \times S^{1}\right)$. It seems to be natural to look in this case, too, for a result parallel to that due to Nenciu [27] and to attempt a derivation of a nontrivial bound on the diffusive growth of energy. But we are aware of only one contribution in this direction made by Barbaroux and Joye [3]; it is based on the general scheme proposed in [23].

In this paper we wish to complete or to strengthen the results from [3] while making use of some ideas from [23]. Thus we aim to consider other classes of time-dependent Hamiltonians whose unperturbed part $H$ has a discrete spectrum with decreasing gaps. In particular, the derived results are applicable to the Howland's model introduced in [20]. In more detail, we deal with a quantum system described by the Hamiltonian $H(t):=H+V(t)$ 
acting on a separable Hilbert space $\mathscr{H}$ and such that $H$ is semibounded and has a pure point spectrum with the spectral decomposition

$$
H=\sum_{n \in \mathbb{N}} E_{n} P_{n}
$$

Assume that the eigen-values $E_{1}<E_{2}<\ldots$ obey the shrinking gap condition

$$
c_{H} \frac{|m-n|}{\max \{m, n\}^{2 \gamma}} \leq\left|E_{m}-E_{n}\right| \leq C_{H} \frac{|m-n|}{\max \{m, n\}^{2 \gamma}}
$$

for some $\gamma \in] 0, \frac{1}{2}$ [ and strictly positive constants $c_{H}, C_{H}$. Notice that condition (1) implies $E_{n} \sim n^{\alpha}$ where $\left.\alpha=1-2 \gamma \in\right] 0,1\left[\right.$ (more precisely, (1) implies that the sequence $E_{n} n^{-\alpha}$ is bounded both from below and from above by strictly positive constants for all sufficiently large $n$ ). To simplify the discussion let us assume, without loss of generality, that $H$ is strictly positive, i.e., $E_{1}>0$.

The time-dependent perturbation $V(t) \in \mathscr{B}(\mathscr{H})$ is supposed to be $T$-periodic and $C^{1}$ in the strong sense. From the strong differentiability it follows that the propagator $U(t, s)$ associated to the Hamiltonian $H+V(t)$ exists and preserves the domain $\operatorname{Dom}(H)$ (see, e.g., [24]).

Let us suppose that $V$ is small with respect to the norm

$$
\|V\|_{p, \gamma}:=\sup _{t \in[0, T]} \sup _{m, n \in \mathbb{N}}\langle m-n\rangle^{p} \max \{m, n\}^{2 \gamma}\left\|V(t)_{m, n}\right\|,
$$

where $p>2$,

$$
\langle m-n\rangle:=\max \{1,|m-n|\},
$$

and $\left\|V(t)_{m, n}\right\|$ denotes the norm of the operator

$$
V(t)_{m, n}:=P_{m} V(t) P_{n}: \operatorname{Ran} P_{n} \rightarrow \operatorname{Ran} P_{m} .
$$

We claim that if, in addition, $\lceil p-1\rceil>1 /(2(1-\alpha))$ then the propagator $U(t, s)$ preserves the form domain $Q_{H}=\operatorname{Dom}\left(H^{1 / 2}\right)$ and for any $\Psi$ from $Q_{H}$ one can estimate the long-time behavior of the energy expectation value by

$$
\langle U(t, 0) \Psi, H U(t, 0) \Psi\rangle=O\left(t^{\sigma}\right), \text { with } \sigma=\frac{2 \alpha}{2\lceil p-1\rceil(1-\alpha)-1}
$$

(more details are given in Theorem 5 below). Here $\lceil x\rceil$ is standing for the ceiling of a real number $x$, i.e., the smallest integer greater than or equal to $x$.

Provided that $[V(t), V(s)]=0$ for every $t, s$ and $\int_{0}^{T} V(t) \mathrm{d} t=0$, the assumption $\|V\|_{p, \gamma} \leq \varepsilon$ can be replaced by $\|V\|_{p+1,0} \leq \varepsilon$, i.e.,

$$
\left\|P_{m} V(t) P_{n}\right\| \leq \frac{\varepsilon}{\langle m-n\rangle^{p+1}} .
$$

The condition $[V(t), V(s)]=0$ is satisfied for example when $V(t)$ is a potential (i.e., a multiplication operator by a function on a certain $L^{2}$ space) or when the time dependence 
of $V(t)$ is factorized, i.e., $V(t)=f(t) v$ where $f(t)$ is a real-valued (T-periodic and $C^{1}$ ) function and $v$ is a time-independent operator on $\mathscr{H}$.

Let us stress that even though the energy diffusion exponent $\sigma$ in (3) can be made arbitrarily small provided $p$ is sufficiently large our result is still far away from the situation when one can prove the dynamical stability in the sense that the energy remains bounded in time for any initial condition [5]. The point is that the time-dependent perturbation $V(t)$ is supposed to be sufficiently regular and small by requiring that $\|V\|_{p, \gamma}<\varepsilon$ where not only the norm but also the positive bound $\varepsilon$ depends on $p$ (see Theorem 5 below for a precise formulation). This plays a role also in the analysis of the Howland's model in Subsection 2.3.1. In this case, $H=|p|^{\alpha}+\varepsilon v(\theta, t)$ and the exponent $\sigma$ in (12) tends to 0 as the order of differentiability of $v(\theta, t)$ in $\theta$, called $k$, tends to infinity. However the coupling constant $\varepsilon$ is supposed to be sufficiently small in dependence on $k$ and so one cannot claim that $\sigma$ equals 0 even if $v(\theta, t)$ is smooth in $\theta$.

On the other hand, to our knowledge, non-trivial examples of time-dependent quantum models for which one can verify this strong type of dynamical stability are rather rare. A periodically time-dependent quadratic Hamiltonian represents such a model. It is explicitly solvable and this is how one can verify the boundedness of energy in the non-resonant case [15]. A broader class of periodically time-dependent models is shown to be dynamically stable for non-resonant values of frequencies with the aid of the KAM (KolmogorovArnold-Moser) type method in [1], see also [14] for some additional discussion. In this connection let us point out a recent example [29] showing that the relationship between the spectral properties of the Floquet operator and the dynamical stability is not so transparent, and it may require a considerable amount of efforts to understand it properly.

Let us compare the result of the current paper, as briefly described above, to the results derived in [23] and [3]. Paper [23] focuses on the general scheme and is not so much concerned with particular cases as that one we are going to deal with here. Nevertheless a possible application to the Howland's classes of perturbations is shortly discussed in Proposition 5.1 and Lemma 5.1. The Howland's classes are determined by a norm which somewhat differs from (2), as explained in more detail in Subsection 2.1. But the difference is not so essential to prevent a comparison. To simplify the discussion let us assume that the eigenvalues of $H$ are simple and behave asymptotically as $E_{n} \sim$ const $n^{\alpha}$, with $0<\alpha<1$. In the particular case when $\|V\|_{p, \gamma}<\infty$ for some $p>1$ and $\gamma=(1-\alpha) / 2$ the bound on the energy diffusion exponent derived in [23] equals $\alpha /\left(2 \gamma-\frac{1}{2}\right)$ provided $\gamma>(1+\alpha) / 4$, i.e., $\alpha<1 / 3$. Our bound $\alpha /\left(2\lceil p-1\rceil \gamma-\frac{1}{2}\right)$, valid for $0<\alpha<1$ and provided $p>2$ and $\lceil p-1\rceil>1 /(4 \gamma)$, is achieved by making use of the rapid decay of matrix entries of $V$ in the direction perpendicular to the diagonal. It follows that we can make the growth of the energy $\langle H\rangle_{\Psi}$ arbitrarily slow by imposing more restrictive assumptions on the perturbation $V$, i.e., by letting the parameter $p$ be sufficiently large.

In paper [3] one treats in fact a larger class of perturbations than we do since one requires only the finiteness of the norm $\|V\|_{p, 0}<\infty$ for $p$ sufficiently large. In other words, no decay of matrix entries of $V$ along the diagonal is supposed. On the other hand, one assumes that the initial quantum state belongs to the domain $\operatorname{Dom}\left(H^{\beta}\right)$ for $\beta$ sufficiently large; $\beta$ is never assumed therein to be smaller than $3 / 2$. Furthermore, there is no assumption on the periodicity of $H(t)$ both in [3] and [23]. On the other hand, our assertion concerns all initial 
states from the domain $\operatorname{Dom}\left(H^{1 / 2}\right)$ but we need a decay of matrix entries of $V$ along the diagonal at least of order $2 \gamma=1-\alpha$. For the sake of comparison let us also recall the bound on the energy diffusion exponent which has been derived in [3]. It is roughly of the form $\alpha /(1-f(p))^{2}$ where $\alpha$ has the same meaning as above, $f(p)$ is positive and $f(p)=O\left(p^{-1}\right)$ as $p \rightarrow \infty$. Hence this bound is never smaller than $\alpha$ and approaches this value as the parameter $p$ tends to infinity.

\section{Upper bound on the energy growth}

\subsection{The gap condition and the modified Howland's classes}

On the contrary to Howland who introduced in [20] the classes $\mathcal{X}(p, \delta)$ equipped with the norm

$$
\|A\|_{p, \delta}^{H}=\sup _{m, n}\left\{(m n)^{\delta}\langle m-n\rangle^{p}\left\|A_{m, n}\right\| ; m, n \geq 1\right\},
$$

we prefer to work with somewhat modified classes, called $\mathcal{Y}(p, \delta)$, whose definition is adjusted to the gap condition (1). Our choice is dictated by an expected asymptotic behavior of eigenvalues of $H$ in a typical situation. Let us briefly explain where condition (1) comes from.

We expect the eigenvalues to behave asymptotically as $E_{n}=$ const $n^{\alpha}(1+o(1))$ where the error term $o(1)$ is supposed to tend to zero sufficiently fast. The spectral gaps $E_{n+1}-E_{n}$ tend to zero as $n \rightarrow \infty$ if $\alpha \in] 0,1$. Keeping the notation $\gamma:=(1-\alpha) / 2$ we wish to estimate the difference $\left|E_{m}-E_{n}\right|$. To this end we replace $E_{n}$ simply by the power sequence $n^{\alpha}$. Then one gets

$$
\frac{m^{\alpha}-n^{\alpha}}{m-n}(m n)^{\gamma}=\frac{\sinh (\alpha y)}{\sinh (y)}=e^{-(1-\alpha)|y|} \frac{1-e^{-2 \alpha|y|}}{1-e^{-2|y|}}
$$

where $e^{2 y}:=m / n$. Since the fraction $\left(1-e^{-2 \alpha|y|}\right) /\left(1-e^{-2|y|}\right)$ can be estimated by positive constants both from above and from below we finally find that

$$
C_{1} \frac{|m-n|}{\max \{m, n\}^{2 \gamma}} \leq\left|m^{\alpha}-n^{\alpha}\right| \leq C_{2} \frac{|m-n|}{\max \{m, n\}^{2 \gamma}}
$$

for some $C_{1}, C_{2}>0$ and all $m, n \in \mathbb{N}$.

Definition 1. Let $p \geq 1, \delta \geq 0$ and $p+2 \delta>1$. We say that an operator $A \in \mathscr{B}(\mathscr{H})$ belongs to the class $\mathcal{Y}(p, \delta)$ if and only if

$$
\|A\|_{p, \delta}:=\sup _{m, n \in \mathbb{N}}\langle m-n\rangle^{p} \max \{m, n\}^{2 \delta}\left\|A_{m, n}\right\|<\infty .
$$

Let $A(t)$ be a $T$-periodic function with values in the space $\mathcal{Y}(p, \delta)$. With some abuse of notation we shall also write

$$
\|A\|_{p, \delta}:=\sup _{t \in[0, T]} \sup _{m, n \in \mathbb{N}}\langle m-n\rangle^{p} \max \{m, n\}^{2 \delta}\left\|A(t)_{m, n}\right\| .
$$


Remarks. (i) It is straightforward to check that $\|\cdot\|_{p, \delta}$ is indeed a norm. Let us note that an equivalent norm is obtained if one replaces $\max \{m, n\}$ by $(m+n)$ in (4).

(ii) Obviously, $\mathcal{Y}(p, \delta) \subset \mathcal{X}(p, \delta)$. Notice that $\mathcal{Y}(p, \delta)$ is a Banach space equipped with the norm $\|\cdot\|_{p, \delta}$.

(iii) For the sake of convenience we have chosen the norm (4) with the restrictions $p \geq$ $1, \delta \geq 0$ and $p+2 \delta>1$ so that if it is finite for a matrix $\left\{A_{m n}\right\}, A_{m n} \in \mathscr{B}\left(\operatorname{Ran} P_{n}, \operatorname{Ran} P_{m}\right)$, then the matrix corresponds to a bounded operator $A \in \mathscr{B}(\mathscr{H})$. Indeed, it is so since one can estimate the operator norm $\|A\|$ by the Shur-Holmgren norm

$$
\|A\|_{S H}:=\max \left\{\sup _{m \in \mathbb{N}} \sum_{n \in \mathbb{N}}\left\|A_{m, n}\right\|, \sup _{n \in \mathbb{N}} \sum_{m \in \mathbb{N}}\left\|A_{m, n}\right\|\right\} .
$$

It clearly holds

$$
\|A\|_{S H} \leq\|A\|_{p, \delta} \sup _{m \in \mathbb{N}} \sum_{n=1}^{\infty} \frac{1}{\langle m-n\rangle^{p} \max \{m, n\}^{2 \delta}} .
$$

The sum on the RHS equals

$$
\begin{aligned}
\frac{1}{m^{2 \delta}}+\sum_{n=1}^{m-1} \frac{1}{(m-n)^{p} m^{2 \delta}}+\sum_{n=m+1}^{\infty} \frac{1}{(n-m)^{p} n^{2 \delta}} & \leq 2+\frac{1}{m^{2 \delta}} \int_{1}^{m} \frac{\mathrm{d} x}{x^{p}}+\sum_{k=1}^{\infty} \frac{1}{k^{p+2 \delta}} \\
& =2+\frac{1-m^{-p+1}}{(p-1) m^{2 \delta}}+\zeta(p+2 \delta) .
\end{aligned}
$$

Setting temporarily $x=\ln (m)$ and $\epsilon=p-1$ one can make use of the inequality

$$
\frac{1}{\epsilon}\left(e^{-2 \delta x}-e^{-(\epsilon+2 \delta) x}\right) \leq \frac{1}{\epsilon+2 \delta}
$$

which is true for all $x \geq 0$ provided $\epsilon \geq 0, \delta \geq 0$ and $\epsilon+2 \delta>0$. Thus one arrives at the estimate

$$
\|A\|_{S H} \leq\left(2+\frac{1}{p+2 \delta-1}+\zeta(p+2 \delta)\right)\|A\|_{p, \delta}
$$

Here $\zeta(u):=\sum_{k=1}^{\infty} k^{-u}$ denotes the Riemann's zeta function.

(iv) Finally let us note that the value $p=\infty$ is admissible. We shall use the norm $\|\cdot\|_{\infty, \delta}$ exclusively in the case of diagonal matrices when it simply reduces to

$$
\|A\|_{\infty, \delta}:=\sup _{n \in \mathbb{N}} n^{2 \delta}\left\|A_{n, n}\right\| .
$$

From Definition 1 one immediately deduces the following lemma.

Lemma 2. Suppose that $H$ is an operator on $\mathscr{H}$ with pure point spectrum whose eigenvalues $E_{1}<E_{2}<\ldots$ obey the upper bound in (1). Let $p>2$. If $A \in \mathcal{Y}(p, \delta)$ then the commutator $[A, H]$ lies in $\mathcal{Y}(p-1, \delta+\gamma)$ and

$$
\|[A, H]\|_{p-1, \delta+\gamma} \leq C_{H}\|A\|_{p, \delta} .
$$


A basic technical tool we need is a lemma concerned with products of two classes $\mathcal{Y}$. For its proof as well as for the remainder of the paper the following two elementary inequalities will be useful. According to the first one, for every $m, k \geq 1$ it holds

$$
\frac{m}{k} \leq 2\langle m-k\rangle \text {. }
$$

In fact, this is a direct consequence of the implication $a, b \geq 1 \Longrightarrow a+b \leq 2 a b$.

The second inequality claims that if $a, b \geq 0$ then

$$
\frac{\langle a+b\rangle}{\langle a\rangle\langle b\rangle} \leq \frac{2}{\langle\min \{a, b\}\rangle} .
$$

This can be reduced to the inequality $\langle 2 a\rangle \leq 2\langle a\rangle$ which is quite obvious.

Lemma 3. Consider two classes $\mathcal{Y}\left(p_{1}, \delta_{1}\right), \mathcal{Y}\left(p_{2}, \delta_{2}\right)$, with $p_{1}, p_{2}>1, \delta_{1}, \delta_{2} \geq 0$. Suppose that numbers $p$, $\delta$ satisfy the inequalities

$$
1<p \leq \min \left\{p_{1}, p_{2}\right\}, \max \left\{\delta_{1}, \delta_{2}\right\} \leq \delta \leq \delta_{1}+\delta_{2}, p+2 \delta \leq \min \left\{p_{1}+2 \delta_{1}, p_{2}+2 \delta_{2}\right\} .
$$

If $A \in \mathcal{Y}\left(p_{1}, \delta_{1}\right)$ and $B \in \mathcal{Y}\left(p_{2}, \delta_{2}\right)$ then

$$
\|A B\|_{p, \delta} \leq C\left(p, \delta-\delta_{0}\right)\|A\|_{p_{1}, \delta_{1}}\|B\|_{p_{2}, \delta_{2}}
$$

where

$$
C(p, \Delta)=2^{p+2 \Delta+1}(1+2 \zeta(p))
$$

and $\delta_{0}=\min \left\{\delta_{1}, \delta_{2}\right\}$. Consequently, $\mathcal{Y}\left(p_{1}, \delta_{1}\right) \mathcal{Y}\left(p_{2}, \delta_{2}\right) \subset \mathcal{Y}(p, \delta)$.

Proof. Under the assumptions we have

$$
\langle m-n\rangle^{p} \max \{m, n\}^{2 \delta}\left\|(A B)_{m n}\right\| \leq\langle m-n\rangle^{p} \max \{m, n\}^{2 \delta} \sum_{\ell=1}^{\infty}\left\|A_{m \ell}\right\|\left\|B_{\ell n}\right\|
$$

which is less than or equal to

$$
\|A\|_{p_{1}, \delta_{1}}\|B\|_{p_{2}, \delta_{2}} \sum_{\ell=1}^{\infty} \frac{\langle m-n\rangle^{p} \max \{m, n\}^{2 \delta}}{\langle m-\ell\rangle^{p_{1}} \max \{m, \ell\}^{2 \delta_{1}}\langle n-\ell\rangle^{p_{2}} \max \{n, \ell\}^{2 \delta_{2}}} .
$$

The summand in (7) can be estimated from above by

$$
\frac{\langle|m-\ell|+|n-\ell|\rangle^{p} \max \{m, n\}^{2 \delta}}{\langle m-\ell\rangle^{p_{1}} \max \{m, \ell\}^{2 \delta_{1}}\langle n-\ell\rangle^{p_{2}} \max \{n, \ell\}^{2 \delta_{2}}} \leq \frac{2^{p}}{\langle\min \{|m-\ell|,|n-\ell|\}\rangle^{p}} h(m, n, \ell)
$$

where

$$
h(m, n, \ell)=\langle m-\ell\rangle^{p-p_{1}}\langle n-\ell\rangle^{p-p_{2}} \frac{\max \{m, n\}^{2 \delta}}{\max \{m, \ell\}^{2 \delta_{1}} \max \{n, \ell\}^{2 \delta_{2}}} .
$$


One can further estimate $h(m, n, \ell)$. For definiteness let us suppose that $m \geq n$. Then, since $p-p_{2} \leq 0$ and $\delta_{2} \geq \delta-\delta_{1}$,

$$
\begin{aligned}
h(m, n, \ell) & \leq\langle m-\ell\rangle^{p-p_{1}} \frac{m^{2 \delta}}{\max \{m, \ell\}^{2 \delta_{1}} \max \{n, \ell\}^{2\left(\delta-\delta_{1}\right)}} \leq\langle m-\ell\rangle^{p-p_{1}}\left(\frac{m}{\ell}\right)^{2\left(\delta-\delta_{1}\right)} \\
& \leq 2^{2\left(\delta-\delta_{1}\right)}\langle m-\ell\rangle^{p-p_{1}+2\left(\delta-\delta_{1}\right)} \leq 2^{2\left(\delta-\delta_{0}\right)} .
\end{aligned}
$$

It follows easily that the sum in (7) is bounded from above by $2^{p+2\left(\delta-\delta_{0}\right)}(2+4 \zeta(p))$ and this estimate implies (6).

Corollary 4. Let $p>2, i \geq 1$ and $\gamma \in] 0, \frac{1}{2}[$. Then the following product formulas hold true:

$$
\begin{aligned}
& \mathcal{Y}(p, i \gamma) \mathcal{Y}(p, i \gamma) \subset \mathcal{Y}(p-1,(i+1) \gamma) \\
& \mathcal{Y}(p,(i-1) \gamma) \mathcal{Y}(p-1, i \gamma) \subset \mathcal{Y}(p-1, i \gamma) \\
& \mathcal{Y}(p+1,(i-1) \gamma) \mathcal{Y}(p-1,(i+1) \gamma) \subset \mathcal{Y}(p-1,(i+1) \gamma)
\end{aligned}
$$

The formulas are also true for the opposite order of factors on the LHS. Moreover, if operators $A$ and $B$ belong to the corresponding classes on the LHS then

$$
\begin{aligned}
& \|A B\|_{p-1,(i+1) \gamma} \leq C_{p}\|A\|_{p, i \gamma}\|B\|_{p, i \gamma} \\
& \|A B\|_{p-1, i \gamma} \leq C_{p}\|A\|_{p,(i-1) \gamma}\|B\|_{p-1, i \gamma} \\
& \|A B\|_{p-1,(i+1) \gamma} \leq 2 C_{p}\|A\|_{p+1,(i-1) \gamma}\|B\|_{p-1,(i+1) \gamma},
\end{aligned}
$$

where

$$
C_{p}:=2^{p+1}(1+2 \zeta(p-1)) .
$$

The norm estimates hold true also for the opposite order of factors $A$ and $B$ in the product.

\subsection{The main theorem}

Theorem 5. Let a quantum system be described by a Hamiltonian of the form

$$
H(t)=H+V(t) \text { on } \mathscr{H}
$$

where $H$ is a self-adjoint operator with a pure point spectrum and the spectral decomposition

$$
H=\sum_{n \in \mathbb{N}} E_{n} P_{n}
$$

Suppose that the eigen-values of $H$ are ordered increasingly and obey the gap condition (1) with $\gamma \in] 0, \frac{1}{2}[$. Set $\alpha=1-2 \gamma$. For $p>2$ assume that

$$
\lceil p-1\rceil>\frac{1}{2(1-\alpha)} .
$$

Then there exists $\varepsilon>0$ such that if $V(t)$ is T-periodic, symmetric, continuously differentiable in the strong sense and obeys $\|V\|_{p, \gamma} \leq \varepsilon$ then the propagator $U(t, s)$ associated 
to the Hamiltonian $H+V(t)$ maps $Q_{H}$, the form domain of $H$, onto itself and for every $\Psi \in Q_{H}$ it holds

$$
\langle H\rangle_{\Psi}(t):=\langle U(t, 0) \Psi, H U(t, 0) \Psi\rangle=O\left(t^{\sigma}\right)
$$

where

$$
\sigma=\frac{2 \alpha}{2\lceil p-1\rceil(1-\alpha)-1}
$$

Remark 6. (i) There is no assumption on the dimension of $\operatorname{Ran} P_{n}$. The multiplicities of eigenvalues may grow arbitrarily, they can even be infinite.

(ii) Suppose that $V(t) \in \mathcal{Y}(p+1,0)$, with $p>2$, is T-periodic, symmetric, continuously differentiable in the strong sense and such that $[V(t), V(s)]=0$ for every $t, s$, and $\bar{V}:=T^{-1} \int_{0}^{T} V(t) \mathrm{d} t=0$. Then one arrives at the same estimate (10). Let us outline the proof.

First, as explained in Remark 12 below in which one has to set $r=p+1, Y=0$, $Z(t)=V(t)$ and $\bar{Z}=0$, one can transform anti-adiabatically $H+V(t)$ into $H+V_{1}(t)$ so that $V_{1}(t) \in \mathcal{Y}(p, \gamma)$ and

$$
\left\|V_{1}\right\|_{p, \gamma} \leq \frac{C_{H}}{2 C_{p+1}}\left(\exp \left(4 C_{p+1} T\|V\|_{p+1,0}\right)-1\right) .
$$

Afterwards one can apply Theorem 5 to the Hamiltonian $H+V_{1}(t)$. Arguing similarly as in the proof of Theorem 5 in Subsection 3.2 one finds that the energy diffusion for the Hamiltonian $H+V_{1}(t)$ is related to that for the Hamiltonian $H+V(t)$ by a quantity which is bounded in time. This is to say that estimate (10) holds true for the time evolution governed by the Hamiltonian $H+V(t)$ as well.

(iii) Provided that $H(t)=H+V(t)$, with $V$ in $C^{1}(\mathbb{R}, \mathscr{B}(\mathscr{H}))$ in the strong sense there exists a trivial bound which does not depend on the spectral properties of $H$ (see [27]), namely

$$
|\langle U(t, 0) \Psi, H(t) U(t, 0) \Psi\rangle| \leq|\langle\Psi, H(0) \Psi\rangle|+|t| \sup _{s \in \mathbb{R}}\|\dot{V}(s)\|\|\Psi\|^{2} .
$$

For its derivation it suffices to notice that

$$
\partial_{t}\langle U(t, 0) \Psi, H(t) U(t, 0) \Psi\rangle=\langle U(t, 0) \Psi, \dot{V}(t) U(t, 0) \Psi\rangle
$$

where $\dot{V}(t)$ denotes the time derivative in the strong sense. The estimate given by Theorem 5 is better than this trivial bound if

$$
\lceil p-1\rceil>p_{\min }:=\frac{2 \alpha+1}{2(1-\alpha)} .
$$

For example, in the case of $\alpha=2 / 3$ (the quantum ball) we get $p_{\min }=7 / 2$. The condition $\lceil p-1\rceil>p_{\text {min }}$ is fulfilled if $p>4$ and then Theorem 5 tells us that $\langle H\rangle_{\Psi}(t)=O\left(t^{4 / 5}\right)$. 
(iv) Apart of the energy itself it is also of interest to consider expectation values of functions of the Hamiltonian $f(H)$ for a suitable choice of the function $f(\lambda)$, see for example [22. Section 3]. In particular this concerns the momenta $H^{m}, m \in \mathbb{N}$. Unfortunately only several steps of our procedure allow for an immediate extension of this type and so we are not able at the moment to deal with this more general case. Let us discuss shortly this point. By inspection of the proofs of Theorem 9 and Lemma 10 one finds that in both of them one can safely replace $H$ by $f(H)$ as long as the sequence $\left\{f\left(E_{n}\right)\right\}$ satisfies, instead of $\left\{E_{n}\right\}$, all assumptions. The propagator $U(t, s)$ in the formulation of Theorem 9 is still associated to the operator $H+W(t)$. The main obstacle is encountered in the proof of Theorem 5 in Subsection 3.2. In analogy to estimate (15) one can derive that

$$
\langle\tilde{U}(t, 0) \tilde{\Psi}, f(H) \tilde{U}(t, 0) \tilde{\Psi}\rangle=\left\langle U(t, 0) \Psi, J(t) f(H) J(t)^{*} U(t, 0) \Psi\right\rangle=O\left(t^{\sigma}\right)
$$

where $\sigma$ is the same as in (15). Here $J(t)$ is, as detailed in the remainder of the paper, a suitable unitary operator constructed with the aid of the anti-adiabatic transform. As a next step in the proof of Theorem 5 one argues that the difference $H-J(t) H J(t)^{*}$ is bounded. However it does not seem to be possible to claim in general the same for the operator $f(H)-J(t) f(H) J(t)^{*}$. And this is exactly the point where the discussed extension fails.

\subsection{Applications}

\subsubsection{The Howland's model}

Let us apply the results of Theorem 5 to the model introduced by Howland in [20] and described by the Hamiltonian $|p|^{\alpha}+\varepsilon v(\theta, t)$, with $\left.\alpha \in\right] 0,1[$, which is supposed to act on $L^{2}\left(S^{1}, \mathrm{~d} \theta\right)$ and to be $2 \pi$-periodic in time. Set $H:=|p|^{\alpha}$. The spectral decomposition of $H$ reads

$$
H=\sum_{n \geq 0} n^{\alpha} P_{n} \text { where } P_{n} \Psi(\theta)=\frac{1}{\pi} \int_{0}^{2 \pi} \cos (n(\theta-s)) \Psi(s) \mathrm{d} s .
$$

Except of the first one the multiplicities of the eigen-values are equal 2. Using integration by parts one derives that any multiplication operator $a$ by a function $a(\theta) \in C^{k}$ obeys the estimate

$$
\left\|P_{m} a P_{n}\right\| \leq \frac{2 \sqrt{2 \pi}\left\|a^{(k)}\right\|}{\langle m-n\rangle^{k}} .
$$

Hence $a \in \mathcal{Y}(k, 0)$. Applying Theorem 5 and Remark 6 ad (iii) we get

Proposition 7. Let $\alpha \in] 0,1[$ and $v(\theta, t)$ be a real-valued function which is $2 \pi$-periodic both in the space and in the time variable. Suppose that $v(\theta, t)$ is $C^{k}$ in $\theta$ and $C^{1}$ in $t$ and such that $\int_{0}^{2 \pi} v(\theta, t) \mathrm{d} t=0$. If $k>3$ and $k>(5-4 \alpha) /(2(1-\alpha))$ then there exists $\varepsilon_{0}>0$ such that for every real $\varepsilon,|\varepsilon|<\varepsilon_{0}$, the propagator $U(t, s)$ associated to

$$
H(t):=|p|^{\alpha}+\varepsilon v(\theta, t) \text { on } L^{2}\left(S^{1}, \mathrm{~d} \theta\right)
$$


preserves the domain $\operatorname{Dom}\left(|p|^{\alpha / 2}\right)$ and for every $\Psi$ from this domain it holds true that

$$
\langle U(t, 0) \Psi, H(t) U(t, 0) \Psi\rangle=O\left(t^{\sigma}\right)
$$

where

$$
\sigma=\frac{2 \alpha}{2(k-2)(1-\alpha)-1} .
$$

Let us summarize that the energy diffusion exponent in the Howland's model can be made arbitrarily small provided the potential on the circle is sufficiently smooth and the coupling constant is sufficiently small.

\subsubsection{Discrete Hamiltonian on the half-line with a slowly growing potential}

Discrete models on a lattice are frequently and intensively studied. Here we are inspired by Example 5.1 in [3]. In contrast to it we restrict ourselves to the usual discrete Laplacian on the half-line rather than considering a long-range Laplacian on the line. To fit the assumptions on which the current paper is based, in particular the gap condition (1), we further restrict ourselves to slowly growing discrete potentials $V(n)=n^{\alpha}$ for some $\alpha, 0<\alpha<1$. Thus we do not cover the most interesting linear case $V(n)=n$.

Set $\mathscr{H}=l^{2}(\mathbb{N})$. Let $\Delta$ be the discrete Laplacian on the half-line,

$$
(\Delta \psi)(1)=\psi(2),(\Delta \psi)(n)=\psi(n-1)+\psi(n+1) \text { for } n \geq 2 .
$$

Further fix a parameter $\alpha, 0<\alpha<1$, and define

$$
(V \psi)(n)=n^{\alpha} \psi(n), \forall n .
$$

Let us consider the Hamiltonian $H(t)=-\Delta+\lambda a(t) V$ where $a(t)$ is a $T$-periodic function, $a \in C^{1}$ and $a(t) \geq a_{0}>0, \forall t \in \mathbb{R}, \lambda>0$ is a coupling constant. Set

$$
b(t)=\lambda \int_{0}^{t} a(s) \mathrm{d} s, \phi(t)=\frac{1}{\lambda a\left(b^{-1}(t)\right)},
$$

and

$$
H_{1}(t)=V-\phi(t) \Delta .
$$

Hence $H(t)=\lambda a(t) H_{1}(b(t))$. Observe that $b(t)=O(t)$ and

$$
b(t+T)=b(t)+\lambda \kappa \text { where } \kappa=\int_{0}^{T} a(s) \mathrm{d} s .
$$

Hence $b^{-1}(t+\lambda \kappa)=b^{-1}(t)+T$. The function $\phi(t)$ is readily seen to be $C^{1}$ and $\lambda \kappa$-periodic. Denote by $U_{1}(t, s)$ the propagator associated to $H_{1}(t)$. Then $U(t, s)=U_{1}(b(t), b(s))$ is the propagator associated to $H(t)$.

Now one can apply Theorem 5 to the Hamiltonian $H_{1}(t)$. The unperturbed part $V$ is diagonal in the standard basis in $l^{2}(\mathbb{N})$, and the eigen-values obey the gap condition (1). On the other hand, the perturbation $-\phi(t) \Delta$ is strongly differentiable and belongs to $\mathcal{Y}(p, 0)$ 
for all $p \geq 1$. Theorem 5 jointly with Remark 6 ad (iii) implies that for any $\sigma>0$ and all initial conditions $\Psi$,

$$
\left\langle U_{1}(t, 0) \Psi, V U_{1}(t, 0) \Psi\right\rangle=O\left(t^{\sigma}\right)
$$

as long as $\lambda \geq \lambda_{0}(\sigma)$ where $\lambda_{0}(\sigma)$ is a lower bound depending on $\sigma$. Replacing $t$ by $b(t)$ one finds that

$$
\langle U(t, 0) \Psi, V U(t, 0) \Psi\rangle=O\left(t^{\sigma}\right) .
$$

\section{Derivation of the main result}

\subsection{Two additional theorems}

The proof of Theorem 5 is based on the following two theorems, Theorem 8 and Theorem 9 . In what follows we use the notation $D:=-\mathrm{i} \partial_{t}$ on the interval $[0, T]$ with the periodic boundary condition.

Theorem 8. Let $K=D+H+V(t)$ be a Floquet Hamiltonian on $L^{2}([0, T], \mathscr{H})$, with $H$ and $V(t)$ satisfying the assumptions of Theorem 5 Let $p>2$ and $q<p-1$ be a natural number. Then there exists $\varepsilon>0$ such that $\|V\|_{p, \gamma} \leq \varepsilon$ implies the existence of a T-periodic family of unitary operators $J(t)$ on $\mathscr{H}$ which is continuously differentiable in the strong sense and such that

$$
K=J(t)(D+H+A+B(t)) J(t)^{*}
$$

where $B(t) \in \mathcal{Y}(p-q,(q+1) \gamma)$ is T-periodic, Hermitian and strongly continuously differentiable, and $A$ is bounded, symmetric and commutes with $H$.

The remainder of the current paper is concerned with the proof of Theorem 8 , Theorem 9 to follow is a mere modification of Proposition 5.1 in [23] in combination with some ideas from [3, Section 2]. This is why we present its proof in a rather sketchy form. Let us also note that the basic idea standing behind the estimates goes back to Nenciu [27].

Theorem 9. Let $H$ be a positive operator with a pure point spectrum and the spectral decomposition $H=\sum_{n} E_{n} P_{n}$. Assume that the eigen-values $0<E_{1}<E_{2}<\ldots$ satisfy $E_{n}=O\left(n^{\alpha}\right)$, with $\alpha>0$. Set $Q_{n}=1-P_{n}$. Let an operator-valued function $W(t) \in$ $\mathscr{B}(\mathscr{H})$ be Hermitian, $C^{1}$ in the strong sense and such that

$$
\forall n \in \mathbb{N}, \quad\left\|P_{n} W(t) Q_{n} H^{-1 / 2}\right\| \leq \frac{\text { const }}{n^{\mu+\frac{\alpha}{2}}}
$$

uniformly in time for some $\mu>1 / 2$. Then the propagator $U(t, s)$ associated with $H+W(t)$ preserves $Q_{H}$, the form domain of $H$, and for every $\Psi$ from $Q_{H}$,

$$
\langle U(t, 0) \Psi, H U(t, 0) \Psi\rangle=O\left(t^{2 \alpha /(2 \mu-1)}\right) .
$$

Remark. The bound on the energy expectation value is nontrivial if $\mu>\frac{1}{2}+\alpha$. 
Proof. Let

$$
W_{d}(t):=\sum_{n=1}^{\infty} P_{n} W(t) P_{n}
$$

be the diagonal part of $W(t)$. It is straightforward to see that $W_{d}(t)$ is again $C^{1}$ in the strong sense. Let $U_{d}(t, s)$ be the propagator associated to $H+W_{d}(t)$. Since $W_{d}(t)$ commutes with $H$ the same if true for $U_{d}(t, s)$. Equivalently this means that $U_{d}(t, s)$ commutes with all projectors $P_{n}$. From the Duhamel's formula we have

$$
R(t):=U(t, 0)-U_{d}(t, 0)=-\mathrm{i} \int_{0}^{t} U_{d}(t, s)\left(W(s)-W_{d}(s)\right) U(s, 0) \mathrm{d} s .
$$

Fix $t>0$ and choose $\Psi \in \operatorname{Dom}(H) \subset \operatorname{Dom}\left(H^{1 / 2}\right)$. Notice that $P_{n}\left(W(s)-W_{d}(s)\right)=$ $P_{n} W(s) Q_{n}$. For any $t^{\prime}, 0 \leq t^{\prime} \leq t$, it holds

$$
\left\|H^{1 / 2} U\left(t^{\prime}, 0\right) \Psi\right\|^{2}=\sum_{n=1}^{\infty} E_{n}\left\|P_{n} U\left(t^{\prime}, 0\right) \Psi\right\|^{2} \leq E_{N}\|\Psi\|^{2}+\sum_{n=N+1}^{\infty} E_{n}\left\|P_{n} U\left(t^{\prime}, 0\right) \Psi\right\|^{2} .
$$

Furthermore,

$$
\left\|P_{n} U\left(t^{\prime}, 0\right) \Psi\right\|^{2} \leq 2\left(\left\|P_{n} \Psi\right\|^{2}+\left\|P_{n} R\left(t^{\prime}\right) \Psi\right\|^{2}\right)
$$

and

$$
\begin{aligned}
\left\|P_{n} R\left(t^{\prime}\right) \Psi\right\| & \leq \int_{0}^{t}\left\|P_{n} W(s) Q_{n} H^{-1 / 2}\right\| \mathrm{d} s \sup _{0 \leq s \leq t}\left\|H^{1 / 2} U(s, 0) \Psi\right\| \\
& \leq \frac{c t}{n^{\mu+\frac{\alpha}{2}}} \sup _{0 \leq s \leq t}\left\|H^{1 / 2} U(s, 0) \Psi\right\| .
\end{aligned}
$$

From these estimates one concludes that for any $t>0$, all $\Psi \in \operatorname{Dom}(H), N \in \mathbb{N}$ and some positive constants $c_{1}, c_{2}$ independent of $t, \Psi$ and $N$ it holds

$$
\left(1-\frac{c_{1} t^{2}}{N^{2 \mu-1}}\right) \sup _{0 \leq s \leq t}\left\|H^{1 / 2} U(s, 0) \Psi\right\|^{2} \leq c_{2} N^{\alpha}\|\Psi\|^{2}+2\left\|H^{1 / 2} \Psi\right\|^{2} .
$$

Setting $N=\left[C t^{2 /(2 \mu-1)}\right]$ where $C>0$ is a sufficiently large constant one deduces that there exists $c_{3}>0$ such that it holds

$$
\left\|H^{1 / 2} U(t, 0) \Psi\right\|^{2} \leq c_{3}\left(t^{2 \alpha /(2 \mu-1)}\|\Psi\|^{2}+\left\|H^{1 / 2} \Psi\right\|^{2}\right)
$$

for all $t \geq 1$ and $\Psi \in \operatorname{Dom}(H)$.

One can extend the validity of (13) to $\Psi \in \operatorname{Dom}\left(H^{1 / 2}\right)$. To this end it suffices to use the fact that $\operatorname{Dom}\left(H^{1 / 2}\right)$ is a Banach space with respect to the norm $\|\Psi\|_{*}=$ $\left(\|\Psi\|^{2}+\left\|H^{1 / 2} \Psi\right\|^{2}\right)^{1 / 2}$, and $\operatorname{Dom}(H) \subset \operatorname{Dom}\left(H^{1 / 2}\right)$ is a dense subspace. Choosing $\Psi \in$ $\operatorname{Dom}\left(H^{1 / 2}\right)$ one can find a sequence $\left\{\Psi_{k}\right\}$ in $\operatorname{Dom}(H)$ such that $\Psi_{k} \rightarrow \Psi$ in $\operatorname{Dom}\left(H^{1 / 2}\right)$. Then (13) implies that $\left\{U(t, 0) \Psi_{k}\right\}$ is a Cauchy sequence in $\operatorname{Dom}\left(H^{1 / 2}\right)$ whose limit necessarily equals $U(t, 0) \Psi$. Hence $\operatorname{Dom}\left(H^{1 / 2}\right)$ is $U(t, 0)$-invariant and (13) is valid also for all $\Psi \in \operatorname{Dom}\left(H^{1 / 2}\right)$. This concludes the proof. 


\subsection{Proof of Theorem 5}

Here we show how Theorem 5 follows from Theorem 8 and Theorem 9

Lemma 10. Assume that $H$ is a positive operator with a pure point spectrum and the spectral decomposition $H=\sum_{n=1}^{\infty} E_{n} P_{n}$, and such that the eigen-values satisfy inf $E_{n} n^{-\alpha}>0$, with $\alpha>0$. Set $Q_{n}=1-P_{n}$. Then for any $p \geq 1$ there exist a constant $c(p, \alpha)>0$ such that for all $\delta>0$,

$$
\forall B \in \mathcal{Y}(p, \delta), \forall n \in \mathbb{N}, \quad\left\|P_{n} B Q_{n} H^{-1 / 2}\right\| \leq c(p, \alpha) \frac{\|B\|_{p, \delta}}{n^{2 \delta+\frac{\alpha}{2}}} .
$$

Proof. Suppose that $B \in \mathcal{Y}(p, \delta)$. By the assumptions, $E_{n} \geq c n^{\alpha}$ for all $n$ and some $c>0$. We have

$$
\left\|P_{n} B Q_{n} H^{-1 / 2}\right\|^{2} \leq \sum_{m, m \neq n} \frac{\left\|B_{n, m}\right\|^{2}}{E_{m}} \leq \frac{1}{c} \sum_{m, m \neq n} \frac{\|B\|_{p, \delta}^{2}}{|m-n|^{2 p} \max \{m, n\}^{4 \delta} m^{\alpha}} .
$$

Now one splits the range of summation in $m$ into three segments: $1 \leq m<n / 2, n / 2 \leq$ $m<n$ and $n<m$. For each case one can apply elementary and rather obvious estimates to show that the expression decays in $n$ at least as $n^{-4 \delta-\alpha}$. In the first case one has to use the fact that $\alpha<1$. We omit the details.

Proof of Theorem 5 Theorem 8 , with $q:=\lceil p-2\rceil$, implies the existence of a transformation

$$
K=J(t)(D+H+A+B(t)) J(t)^{*}
$$

where $A$ is bounded and diagonal and $B(t) \in \mathcal{Y}(p-q,(q+1) \gamma)$. Since $p>2$ and $q=\lceil p-2\rceil$ we have $q \geq 1$ and $p-q>1$. Set $W(t):=A+B(t)$. Then $P_{n} W(t) Q_{n}=P_{n} B(t) Q_{n}$. The gap condition (1) guarantees that the assumptions of Lemma 10 are satisfied and thus one finds that

$$
\left\|P_{n} W(t) Q_{n} H^{-1 / 2}\right\|=\left\|P_{n} B(t) Q_{n} H^{-1 / 2}\right\| \leq \mathrm{const} \cdot n^{-\mu-\frac{\alpha}{2}},
$$

with $\mu=2(q+1) \gamma=\lceil p-1\rceil(1-\alpha)$. Notice that assumption (9) means that $\mu>1 / 2$. In virtue of Theorem 9 , the propagator $\tilde{U}(t, s)$ associated to $H+W(t)$ maps the form domain $Q_{H}$ onto itself and fulfills

$$
\langle\tilde{U}(t, 0) \tilde{\Psi}, H \tilde{U}(t, 0) \tilde{\Psi}\rangle=O\left(t^{\sigma}\right), \text { with } \sigma=\frac{2 \alpha}{2\lceil p-1\rceil(1-\alpha)-1},
$$

for every $\tilde{\Psi} \in Q_{H}$.

Equality (14) implies that

$$
H+V(t)=J(t) H J(t)^{*}+\mathrm{i} \dot{J}(t) J(t)^{*}+J(t) W(t) J(t)^{*} .
$$

Since the family $J(t)$ is known to be continuously differentiable in the strong sense it follows from the uniform boundedness principle that the derivative $\dot{J}(t)$ is a bounded operator. Moreover, using the periodicity and applying the uniform boundedness principle 
once more one finds that $\|\dot{J}(t)\|$ is bounded uniformly in $t$. Hence all operators occurring in equality (16), except of $H$, are bounded. One deduces from (16) that $J(t)$ maps Dom $H$ onto itself for every $t$ and that the same is also true for the form domain. Set $U(t, s):=J(t) \tilde{U}(t, s) J(s)^{*}$. Then $U(t, s)$ is the propagator corresponding to $H+V(t)$. For any $\Psi \in Q_{H}$ we have

$$
\begin{aligned}
\langle H\rangle_{\Psi}(t) & =\langle U(t, 0) \Psi, H U(t, 0) \Psi\rangle=\left\langle U(t, 0) \Psi, J(t) H J(t)^{*} U(t, 0) \Psi\right\rangle+O(1) \\
& =\langle\tilde{U}(t, 0) \tilde{\Psi}, H \tilde{U}(t, 0) \tilde{\Psi}\rangle+O(1)=O\left(t^{\sigma}\right)
\end{aligned}
$$

where $\tilde{\Psi}:=J(0)^{*} \Psi$. This proves the theorem.

\subsection{The idea of the proof of Theorem 8}

It remains to prove Theorem 8 . The proof is somewhat lengthy and the remainder of the paper is devoted to it. Let us explain the main idea. The proof combines the anti-adiabatic transformation due to Howland (see Section 4) with a (properly modified) diagonalization method, as presented in [13] (see Section 5). This procedure is applied repeatedly until achieving the required properties of the perturbation. Let us describe one step in this approach when starting from the Floquet Hamiltonian

$$
K_{\triangle}:=D+H+Y+Z(t)
$$

where $Y \in \mathcal{Y}(\infty, \gamma)$ is Hermitian and diagonal (i.e., commuting with $H$ ) and $Z(t) \in$ $\mathcal{Y}(r, i \gamma)$ is symmetric, $T$-periodic and strongly $C^{1}$. The parameters are supposed to satisfy $i \geq 1, r>2$.

Firstly, using the anti-adiabatic transform we try to improve the decay of entries of $Z(t)$ along the main diagonal when paying for it by a worse decay of elements in the direction perpendicular to the diagonal. In more detail, we would like to transform $Z(t) \in \mathcal{Y}(r, i \gamma)$ into $Z_{\diamond}(t) \in \mathcal{Y}(r-1,(i+1) \gamma)$. Unfortunately, we are not able to get rid of the extra term $\bar{Z} \in \mathcal{Y}(r, i \gamma)$, the time average of $Z(t)$. The anti-adiabatic transform can be schematically described as

$$
K_{\triangle}=D+H+Y+Z(t) \rightarrow K_{\diamond}=D+H+Y+\bar{Z}+Z_{\diamond}(t) .
$$

To cope with the unwanted extra term we apply afterwards a diagonalization procedure which in fact means the transform

$$
K_{\diamond}=D+H+Y+\bar{Z}+Z_{\diamond}(t) \rightarrow K_{\diamond}:=D+H+A+B(t)
$$

where $A$ and $B(t)$ already have the desired properties, i.e., $B(t) \in \mathcal{Y}(r-1,(i+1) \gamma)$ is symmetric, $T$-periodic and strongly $C^{1}$, and $A \in \mathcal{Y}(\infty, \gamma)$ is Hermitian and commuting with $H$.

\section{The anti-adiabatic transform}

In this section we adapt the strategy of Howland [20] and make precise the mapping $K_{\triangle} \rightarrow$ $K_{\diamond}$, as announced in Subsection 3.3. Using the anti-adiabatic transform, i.e., roughly speaking, by applying the commutator with $H$ one can improve the decay of matrix entries of the 
perturbation along the main diagonal at the expense of a slower decay in the direction perpendicular to the diagonal. Using the language of classes $\mathcal{Y}(p, \delta)$, the anti-adiabatic transform may be viewed as passing from a perturbation $Z(t) \in \mathcal{Y}(p, \delta)$ to a new perturbation $Z_{1}(t) \in \mathcal{Y}(p-1, \delta+\gamma)$ where $\gamma$ comes from the gap condition (1) (see Lemma 2).

Let us introduce the transform in detail. Let $K_{\triangle}$ be a Floquet Hamiltonian of the form

$$
K_{\triangle}=D+H+Y+Z(t),
$$

with $H$ satisfying the assumptions of Theorem 5, $Y \in \mathcal{Y}(\infty, \gamma)$ being Hermitian and commuting with $H$, and $Z(t) \in \mathcal{Y}(r, i \gamma)$ being Hermitian, $T$-periodic and continuous in the strong sense. By the uniform boundedness principle, $\|Z(t)\|$ is bounded uniformly in $t$. The parameters are supposed to satisfy $r>2, i \geq 1$. Set

$$
\bar{Z}:=\frac{1}{T} \int_{0}^{T} Z(t) \mathrm{d} t, \quad \tilde{Z}(t)=Z(t)-\bar{Z} .
$$

Define

$$
F(t):=\int_{0}^{t} \tilde{Z}(s) \mathrm{d} s
$$

so that $F(t)$ is Hermitian, $T$-periodic, strongly $C^{1}$ and lying in $\mathcal{Y}(r, i \gamma)$. Let us define $K_{\diamond}$ by the gauge-type transformation of $K_{\triangle}$,

$$
K_{\diamond}:=e^{\mathrm{i} F(t)} K_{\triangle} e^{-\mathrm{i} F(t)}=D+H+Y+\bar{Z}+Z_{\diamond}(t)
$$

with

$$
Z_{\diamond}(t)=e^{\mathrm{i} F(t)}(D+H+Y+Z(t)) e^{-\mathrm{i} F(t)}-(D+H+Y+\bar{Z}) .
$$

The main result related to the anti-adiabatic transform is as follows.

Proposition 11. Let $r>2, i \geq 1, \gamma \in] 0, \frac{1}{2}$ [, and $H$ be a self-adjoint operator with a pure point spectrum and the spectral decomposition $H=\sum_{n} E_{n} P_{n}$. Assume that the eigenvalues $\left\{E_{n}\right\}_{n=1}^{\infty}$ are ordered increasingly and satisfy the inequality

$$
\left|E_{m}-E_{n}\right| \leq C_{H} \frac{|m-n|}{\max \{m, n\}^{2 \gamma}}
$$

Furthermore, $Y$ and $Z(t)$ obey the assumptions formulated above.

Then $Z_{\diamond}(t)$ defined in (17) is T-periodic, continuous in the strong sense, Hermitian, and lies in $\mathcal{Y}(r-1,(i+1) \gamma)$. The norm of $Z_{\diamond}$ obeys the bound

$$
\left\|Z_{\diamond}\right\|_{r-1,(i+1) \gamma} \leq \frac{\exp \left(4 C_{r} T\|Z\|_{r, i \gamma}\right)-1}{2 C_{r}}\left(C_{H}+4\|Y\|_{\infty, \gamma}+2 C_{r}\|Z\|_{r, i \gamma}\right),
$$

with the constant $C_{r}$ defined in (8). The operator-valued function $e^{\mathrm{i} F(t)}$ is $C^{1}$ in the strong sense. Moreover, if $Z(t)$ is $C^{1}$ in the strong sense then the same is true for $Z_{\diamond}(t)$. 
Proof. The periodicity and the differentiability are clear from the above discussion. The RHS of (17) can be expanded according to the formula

$$
e^{A} B e^{-A}=B+\sum_{j=1}^{\infty} \frac{1}{j !} \operatorname{ad}_{A}^{j}(B) .
$$

Here we use the notation $\operatorname{ad}_{A}(B):=[A, B]=A B-B A$. Since $\operatorname{ad}_{F(t)} D=\mathrm{i} \dot{F}(t)=\mathrm{i} \tilde{Z}(t)$ we get

$$
\begin{aligned}
Z_{\diamond}(t) & =\sum_{j=1}^{\infty} \frac{\mathrm{i}^{j}}{j !} \operatorname{ad}_{F(t)}^{j-1}(\mathrm{i} \tilde{Z}(t)+[F(t), H+Y+Z(t)])+\tilde{Z}(t) \\
& =\sum_{j=1}^{\infty} \frac{\mathrm{i}^{j}}{j !} \operatorname{ad}_{F(t)}^{j-1} X(t)
\end{aligned}
$$

where

$X(t):=\operatorname{ad}_{F(t)}\left(H+Y+Z(t)-\frac{1}{j+1} \tilde{Z}(t)\right)=\operatorname{ad}_{F(t)}\left(H+Y+\frac{j}{j+1} Z(t)+\frac{1}{j+1} \bar{Z}\right)$.

By Lemma 2, $\operatorname{ad}_{F(t)} H \in \mathcal{Y}(r-1,(i+1) \gamma)$, and according to Corollary 4, the same holds true for $\operatorname{ad}_{F(t)} Z(t)$ and $\operatorname{ad}_{F(t)} \bar{Z}$. Notice also that $\|\bar{Z}\|_{p, \delta} \leq\|Z\|_{p, \delta}$. Furthermore, since $Y \in \mathcal{Y}(\infty, \gamma)$ is diagonal we have

$$
\begin{aligned}
& \langle m-n\rangle^{r-1} \max \{m, n\}^{2(i+1) \gamma}\left\|(F(t) Y)_{m, n}\right\| \\
& \leq \frac{1}{\langle m-n\rangle}\left(\frac{\max \{m, n\}}{n}\right)^{2 \gamma} n^{2 \gamma}\|F\|_{r, i \gamma}\left\|Y_{n, n}\right\| \leq 2^{2 \gamma}\|F\|_{r, i \gamma}\|Y\|_{\infty, \gamma} .
\end{aligned}
$$

Hence $\|F(t) Y\|_{r-1,(i+1) \gamma} \leq 2\|F\|_{r, i \gamma}\|Y\|_{\infty, \gamma}$. The same estimate is true for $\|Y F(t)\|_{r-1,(i+1) \gamma}$ and therefore $\left\|\operatorname{ad}_{F} Y\right\|_{r-1,(i+1) \gamma} \leq 4\|F\|_{r, i \gamma}\|Y\|_{\infty, \gamma}$. We conclude that $X(t)$ belongs to $\mathcal{Y}(r-1,(i+1) \gamma)$ and

$$
\|X\|_{r-1,(i+1) \gamma} \leq\|F\|_{r, i \gamma}\left(C_{H}+4\|Y\|_{\infty, \gamma}+2 C_{r}\|Z\|_{r, i \gamma}\right) .
$$

Recalling Corollary 4 once more we have

$$
\mathcal{Y}(r-1,(i+1) \gamma) \mathcal{Y}(r, i \gamma), \mathcal{Y}(r, i \gamma) \mathcal{Y}(r-1,(i+1) \gamma) \subset \mathcal{Y}(r-1,(i+1) \gamma)
$$

and so $\operatorname{ad}_{F(t)}^{j-1} X(t)$ lies in $\mathcal{Y}(r-1,(i+1) \gamma)$ as well and

$$
\left\|\operatorname{ad}_{F}^{j-1} X\right\|_{r-1,(i+1) \gamma} \leq\left(2 C_{r}\|F\|_{r, i \gamma}\right)^{j-1}\|X\|_{r-1,(i+1) \gamma} .
$$

Consequently, the series (19) converges in the Banach space $\mathcal{Y}(r-1,(i+1) \gamma)$. To derive inequality (18) from (20) and (21) one applies the estimate $\|F\|_{r, i \gamma} \leq 2 T\|Z\|_{r, i \gamma}$ which immediately follows from the definition of $F(t)$ and $\tilde{Z}(t)$. This completes the proof.

Remark 12. The proposition holds also true for $i=0$ provided $[Z(t), Z(s)]=0$ for every $t, s$. In this case $F(t)$ commutes with $Z(t)$ and $\bar{Z}$, and the formula (19) holds true with $X(t)=\operatorname{ad}_{F(t)}(H+Y)$. Repeating the steps from the proof of the proposition one arrives at the inequality

$$
\left\|Z_{\diamond}\right\|_{r-1,(i+1) \gamma} \leq \frac{\exp \left(4 C_{r} T\|Z\|_{r, i \gamma}\right)-1}{2 C_{r}}\left(C_{H}+2\|Y\|_{\infty, \gamma}\right) .
$$




\section{The diagonalization procedure}

\subsection{Formulation of the result}

The main result of this section is formulated in the following proposition.

Proposition 13. Let $i \geq 1$ be a natural number, $\gamma \in] 0, \frac{1}{2}[$, and $H$ be a self-adjoint operator with a pure point spectrum and the spectral decomposition $H=\sum_{n} E_{n} P_{n}$. Assume that the eigen-values $\left\{E_{n}\right\}_{n=1}^{\infty}$ are ordered increasingly and satisfy the inequality

$$
\left|E_{m}-E_{n}\right| \geq c_{H} \frac{|m-n|}{\max \{m, n\}^{2 \gamma}} .
$$

Let $Y \in \mathcal{Y}(\infty, \gamma)$ be Hermitian and commuting with $H$. Suppose that $\bar{Z}$ is Hermitian and belongs to the class $\mathcal{Y}(r, i \gamma)$ for some $r>2$. Finally, assume that

$$
\|Y\|_{\infty, \gamma}+\|\bar{Z}\|_{r, i \gamma} \leq \frac{c_{H}}{4 \pi C_{r+1}}
$$

with the constant $C_{r+1}$ given by (8).

Then there exists $U$, a unitary operator on $\mathscr{H}$, such that

$$
U(H+Y+\bar{Z}) U^{*}=H+A
$$

where $A \in \mathcal{Y}(\infty, \gamma)$ commutes with $H$ and obeys

$$
\|A\|_{\infty, \gamma} \leq 2\left(\|Y\|_{\infty, \gamma}+\|\bar{Z}\|_{r, i \gamma}\right) .
$$

Moreover, for every operator $X \in \mathcal{Y}(r-1,(i+1) \gamma)$ it holds

$$
\left\|U X U^{*}\right\|_{r-1,(i+1) \gamma} \leq \exp \left(2 \frac{C_{r}}{C_{r+1}}\right)\|X\|_{r-1,(i+1) \gamma} .
$$

Since $U$ does not depend on time this result can be interpreted in the following way.

Corollary 14. Let us consider a Floquet Hamiltonian of the form

$$
K_{\diamond}=D+H+Y+\bar{Z}+Z_{\diamond}(t)
$$

where $H, Y$ and $\bar{Z}$ obey the same assumptions as in Proposition 13 with $r>2$ and $i \geq 1$, and $Z_{\diamond}(t) \in \mathcal{Y}(r-1,(i+1) \gamma)$ is T-periodic, continuously differentiable in the strong sense and Hermitian.

Then there exists a unitary operator $U$ on $\mathscr{H}$ such that for the transformed Floquet Hamiltonian

$$
K_{\diamond}:=U K_{\diamond} U^{*}=D+H+A+B(t)
$$

it holds: $A \in \mathcal{Y}(\infty, \gamma)$ commutes with $H$ and fulfills (25),

$$
B(t):=U Z_{\diamond}(t) U^{*} \in \mathcal{Y}(r-1,(i+1) \gamma)
$$

is T-periodic, continuously differentiable in the strong sense, Hermitian and satisfies

$$
\|B\|_{r-1,(i+1) \gamma} \leq \exp \left(2 \frac{C_{r}}{C_{r+1}}\right)\left\|Z_{\diamond}\right\|_{r-1,(i+1) \gamma} .
$$

The proof of Proposition 13 is a modification (to the case of shrinking gaps) of a diagonalization procedure introduced in [13] and conventionally called the progressive diagonalization method. 


\subsection{The algorithm}

The diagonalization procedure is constructed iteratively, let us first describe the algorithm. Starting from $H+Y+\bar{Z}$ we construct the first 4-tuple of operators

$$
U_{0}:=1, G_{1}:=Y+\operatorname{diag} \bar{Z}, V_{1}:=\operatorname{offdiag} \bar{Z}, H_{1}:=H+G_{1}+V_{1},
$$

where

$$
\operatorname{diag} X:=\sum_{n \in \mathbb{N}} P_{n} X P_{n}, \quad \text { offdiag } X:=\sum_{m \neq n} P_{m} X P_{n}
$$

denote the diagonal and the off diagonal part of the matrix of an operator $X$ with respect to the eigen-basis of $H$. We define recursively a sequence of operators $H_{s}, G_{s}, V_{s}, W_{s}$ and $U_{s}$ by the following rules: provided $G_{s}$ and $V_{s}$ have been already defined let $W_{s}$ be the solution of

$$
\left[H+G_{s}, W_{s}\right]=V_{s} \text { and } \operatorname{diag} W_{s}=0
$$

We define

$$
H_{s+1}:=e^{W_{s}} H_{s} e^{-W_{s}}
$$

Finally, we set

$$
U_{s}:=e^{W_{s}} U_{s-1}, G_{s+1}:=\operatorname{diag} H_{s+1}-H, V_{s+1}:=\text { offdiag } H_{s+1} .
$$

Since $H_{s}=H+G_{s}+V_{s}$ for all $s$ and with the aid of (27) one derives from (28) that

$$
\begin{aligned}
H_{s+1} & =H_{s}+\sum_{k=1}^{\infty} \frac{1}{k !} \operatorname{ad}_{W_{s}}^{k-1}\left[W_{s}, H_{s}\right]=H+G_{s}+V_{s}+\sum_{k=1}^{\infty} \frac{1}{k !} \operatorname{ad}_{W_{s}}^{k-1}\left(-V_{s}+\left[W_{s}, V_{s}\right]\right) \\
& =H+G_{s}+\Phi\left(\operatorname{ad}_{W_{s}}\right) V_{s}
\end{aligned}
$$

where

$$
\Phi(x):=\sum_{k=1}^{\infty} \frac{k}{(k+1) !} x^{k}=e^{x}-\frac{1}{x}\left(e^{x}-1\right)
$$

Observe also that in the course of the algorithm, $G_{s}$ is always diagonal (commuting with $H$ ) and symmetric, $V_{s}$ is symmetric and off diagonal, $W_{s}$ is antisymmetric and off diagonal. Therefore $e^{W_{s}}$ and $U_{s}$ are unitary. It is straightforward to prove by induction that for every $s=1,2, \ldots$,

$$
H+G_{s+1}+V_{s+1}=U_{s}(H+Y+\bar{Z}) U_{s}^{*} .
$$

\subsection{Auxiliary facts}

To solve the commutator equation (27) we need the following result taken from a paper by Bhatia and Rosenthal.

Lemma 15 ([6]). Let $E$ and $F$ be two Hilbert spaces. Let $A$ and $B$ be Hermitian operators (i.e., bounded and self-adjoint) on $E$ and $F$, respectively, such that $\operatorname{dist}(\sigma(A), \sigma(B))>0$. 
Then for every bounded operator $Y: F \rightarrow E$ there exists a unique bounded operator $X: F \rightarrow$ E such that

$$
A X-X B=Y \text {. }
$$

Moreover, the inequality

$$
\|X\| \leq \frac{\pi}{2 \operatorname{dist}(\sigma(A), \sigma(B))}\|Y\|,
$$

holds true.

Remark. The solution $X$ is given by

$$
X=\int_{\mathbb{R}} e^{-\mathrm{i} t A} Y e^{\mathrm{i} t B} f(t) \mathrm{d} t
$$

for any $f \in L^{1}(\mathbb{R})$ such that its Fourier image obeys $\hat{f}(s)=1 / \sqrt{2 \pi} s$ on the set $\sigma(A)-\sigma(B)$. This implies $\|X\| \leq\|f\|_{1}\|Y\|$, and optimizing over such $f$ one gets the constant $\pi / 2$.

In the algorithm plays a certain role the function $\Phi(x)$ introduced in (31). It is supposed to be defined on the interval $[0, \infty[$. Let us point out here some of its elementary properties. This is a strictly increasing function mapping the interval $[0, \infty[$ onto itself. It holds $\Phi(0)=$ $0, \Phi(1)=1$, and so the function maps also the interval ] $0,1[$ onto itself. Moreover, $\Phi(x)$ is a convex function and so

$$
\forall x \in] 0,1[, \Phi(x)<x .
$$

Further, let us consider a sequence $\left\{x_{s}\right\}_{s=1}^{\infty}$ formed by nonnegative numbers obeying the inequalities

$$
\forall s \in \mathbb{N}, x_{s+1} \leq \Phi\left(x_{s}\right) x_{s} .
$$

If $x_{1}<1$ then the sequence is non-increasing and (33), (34) imply that $x_{s+1} \leq x_{s}^{2}$. It follows that

$$
\forall s \in \mathbb{N}, x_{s} \leq x_{1}^{2^{s-1}}
$$

and

$$
\sum_{s=1}^{\infty} x_{s} \leq \frac{x_{1}}{1-x_{1}}<\infty
$$

\subsection{Convergence of the algorithm}

Proof of Proposition 13. We have to prove that $V_{s} \rightarrow 0, G_{s} \rightarrow A$ and $U_{s} \rightarrow U$. The key ingredient of the algorithm is the control of the size of $W_{s}$ given as the off diagonal solution to the commutator equation (27). For every $m \neq n$ we seek $W_{s}(m, n)$ such that

$$
\left(E_{m}+\left(G_{s}\right)_{m, m}\right)\left(W_{s}\right)_{m, n}-\left(W_{s}\right)_{m, n}\left(E_{n}+\left(G_{s}\right)_{n, n}\right)=\left(V_{s}\right)_{m, n} .
$$

Suppose for the moment that $G_{s}$ lies in $\mathcal{Y}(\infty, \gamma)$ for every $s \in \mathbb{N}$ with

$$
\left\|G_{s}\right\|_{\infty, \gamma} \leq \frac{c_{H}}{6}
$$


The norm $\|\cdot\|_{\infty, \gamma}$ makes sense in this case since $G_{s}$ is diagonal for every $s \in \mathbb{N}$. The spectrum of $E_{n}+\left(G_{s}\right)_{n, n}$ is a subset of the interval

$$
\left[E_{n}-\frac{\left\|G_{s}\right\|_{\infty, \gamma}}{n^{2 \gamma}}, E_{n}+\frac{\left\|G_{s}\right\|_{\infty, \gamma}}{n^{2 \gamma}}\right] .
$$

Owing to (22) the distance between the spectrum of $E_{m}+\left(G_{s}\right)_{m, m}$ and $E_{n}+\left(G_{s}\right)_{n, n}$ can be estimated from below by

$$
\begin{aligned}
\left|E_{m}-E_{n}\right|-\left\|G_{s}\right\|_{\infty, \gamma}\left(m^{-2 \gamma}+n^{-2 \gamma}\right) & \geq c_{H} \frac{|m-n|}{\max \{m, n\}^{2 \gamma}}-\frac{c_{H}}{6}\left(m^{-2 \gamma}+n^{-2 \gamma}\right) \\
& \geq \frac{c_{H}|m-n|}{2 \max \{m, n\}^{2 \gamma}}
\end{aligned}
$$

The last inequality in (37) is a consequence of the following estimate where we assume for definiteness that $m>n$ (recall that $2 \gamma<1)$ :

$$
\frac{3(m-n)}{m^{2 \gamma}} \geq m^{-2 \gamma}+\frac{m}{n} m^{-2 \gamma} \geq m^{-2 \gamma}+n^{-2 \gamma} .
$$

Applying Lemma[15 we conclude that

$$
\left\|\left(W_{s}\right)_{m, n}\right\| \leq \frac{\pi \max \{m, n\}^{2 \gamma}}{c_{H}|m-n|}\left\|\left(V_{s}\right)_{m, n}\right\| .
$$

Set

$$
M:=\frac{c_{H}}{2 \pi C_{r+1}}, \quad x_{s}:=\frac{\left\|V_{s}\right\|_{r, i \gamma}}{M},
$$

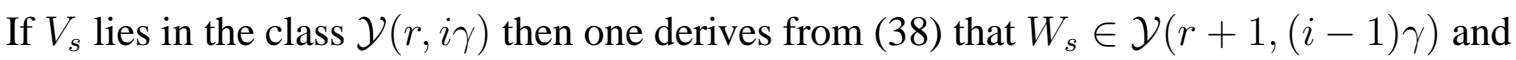

$$
\left\|W_{s}\right\|_{r+1,(i-1) \gamma} \leq \frac{\pi}{c_{H}}\left\|V_{s}\right\|_{r, i \gamma}=\frac{x_{s}}{2 C_{r+1}} .
$$

From Corollary 4 it follows that $\operatorname{ad}_{W_{s}}^{k} V_{s} \in \mathcal{Y}(r, i \gamma)$ and

$$
\left\|\operatorname{ad}_{W_{s}}^{k} V_{s}\right\|_{r, i \gamma} \leq\left(2 C_{r+1}\left\|W_{s}\right\|_{r+1,(i-1) \gamma}\right)^{k}\left\|V_{s}\right\|_{r, i \gamma} \leq x_{s}^{k}\left\|V_{s}\right\|_{r, i \gamma}
$$

Since $V_{s+1}$ is defined as the off diagonal part of $H_{s+1}$ we get from (30) and (41) that

$$
V_{s+1}=\operatorname{offdiag}\left(\Phi\left(\operatorname{ad}_{W_{s}}\right) V_{s}\right) .
$$

and so

$$
\left\|V_{s+1}\right\|_{r, i \gamma} \leq \Phi\left(x_{s}\right)\left\|V_{s}\right\|_{r, i \gamma} .
$$

Hence the sequence $\left\{x_{s}\right\}$ defined in (39) fulfills inequalities (34).

Since $\left\|V_{1}\right\|_{r, i \gamma} \leq\|\bar{Z}\|_{r, i \gamma}$ assumption (23) implies $x_{1} \leq 1 / 2$. We know from the discussion at the end of Subsection 5.3 that in that case the series $\sum x_{s}$ is convergent. It follows that $\left\|V_{s}\right\|_{r, i \gamma} \rightarrow 0$ and, using the estimate

$$
\left\|W_{s}\right\| \leq\left\|W_{s}\right\|_{S H} \leq(1+2 \zeta(r+1))\left\|W_{s}\right\|_{r+1,(i-1) \gamma}
$$


and (40), also that $U_{s}$ converges to a unitary operator $U$ in $\mathscr{B}(\mathscr{H})$. Furthermore, from (30) and (29) one deduces that

$$
G_{s+1}-G_{s}=\operatorname{diag}\left(\Phi\left(\operatorname{ad}_{W_{s}}\right) V_{s}\right) .
$$

Since $G_{s}$ is diagonal and $i \geq 1$ we have

$$
\left\|G_{s+1}-G_{s}\right\|_{\infty, \gamma}=\left\|G_{s+1}-G_{s}\right\|_{r, \gamma} \leq\left\|G_{s+1}-G_{s}\right\|_{r, i \gamma} \leq\left\|\Phi\left(\operatorname{ad}_{W_{s}}\right) V_{s}\right\|_{r, i \gamma} .
$$

Using once more (40) and (41) one finds that

$$
\left\|G_{s+1}-G_{s}\right\|=\left\|G_{s+1}-G_{s}\right\|_{\infty, 0} \leq\left\|G_{s+1}-G_{s}\right\|_{\infty, \gamma} \leq M \Phi\left(x_{s}\right) x_{s} .
$$

From here one concludes that $\left\{G_{s}\right\}$ is a Cauchy sequence both in $\mathcal{Y}(\infty, \gamma)$ and $\mathscr{B}(\mathscr{H})$. Hence $G_{s}$ converges to a diagonal operator $A$ which lies in $\mathcal{Y}(\infty, \gamma)$.

We must verify that condition (36) is actually fulfilled. Observe from (8) that $C_{p}>2^{3} \cdot 3$ if $p>2$. By the assumptions,

$$
\left\|G_{1}\right\|_{\infty, \gamma} \leq\|Y\|_{\infty, \gamma}+\|\bar{Z}\|_{r, i \gamma}<\frac{c_{H}}{12} .
$$

Furthermore, from (42) it follows that

$$
\left\|G_{s+1}\right\|_{\infty, \gamma} \leq\left\|G_{1}\right\|_{\infty, \gamma}+\sum_{j=1}^{s}\left\|G_{s+1}-G_{s}\right\|_{\infty, \gamma} \leq \frac{c_{H}}{12}+M \sum_{j=1}^{\infty} x_{j} \Phi\left(x_{j}\right) .
$$

Recalling that $x_{1} \leq 1 / 2$ one gets

$$
M \sum_{j=1}^{\infty} x_{j} \Phi\left(x_{j}\right) \leq \frac{M x_{1}^{2}}{1-x_{1}} \leq M x_{1} \leq\|\bar{Z}\|_{r, i \gamma}<\frac{c_{H}}{12} .
$$

The last inequality is again a consequence of assumption (23). One concludes that condition (36) is fulfilled for all $s$.

Since all operators occurring in (32) except of $H$ are bounded one deduces from this equality that $U_{s}$ preserves the domain of $H$ for all $s$. Since $H$ is a closed operator the limit in equality (32), as $s \rightarrow \infty$, can be carried out and results in equality (24).

From the computations in (43), (44) it also follows that

$$
\left\|G_{s+1}\right\|_{\infty, \gamma} \leq\left\|G_{1}\right\|_{\infty, \gamma}+M x_{1}=\left\|G_{1}\right\|_{\infty, \gamma}+\left\|V_{1}\right\|_{r, i \gamma} \leq\|Y\|_{\infty, \gamma}+2\|\bar{Z}\|_{r, i \gamma} .
$$

Sending $s$ to infinity one verifies the estimate (25). Furthermore, estimate (40) implies

$$
\sum_{s=1}^{\infty}\left\|W_{s}\right\|_{r+1,(i-1) \gamma} \leq \frac{1}{2 C_{r+1}} \sum_{s=1}^{\infty} x_{s} \leq \frac{x_{1}}{2 C_{r+1}\left(1-x_{1}\right)} \leq \frac{1}{2 C_{r+1}} .
$$

From Corollary 4 we deduce that the operator $\operatorname{ad}_{W_{s}}$ is well defined on the Banach space $\mathcal{Y}(r-1,(i+1) \gamma)$, with a norm bounded from above by $4 C_{r}\left\|W_{s}\right\|_{r+1,(i-1) \gamma}$. Thus for 
$X \in \mathcal{Y}(r-1,(i+1) \gamma)$ one can estimate

$$
\begin{aligned}
\left\|U X U^{*}\right\|_{r-1,(i+1) \gamma} & =\lim _{s \rightarrow \infty}\left\|e^{W_{s}} e^{W_{s-1}} \cdots e^{W_{1}} X e^{-W_{1}} \cdots e^{-W_{s-1}} e^{-W_{s}}\right\|_{r-1,(i+1) \gamma} \\
& \leq \exp \left(4 C_{r} \sum_{s=1}^{\infty}\left\|W_{s}\right\|_{r+1,(i-1) \gamma}\right)\|X\|_{r-1,(i+1) \gamma} \\
& \leq \exp \left(2 \frac{C_{r}}{C_{r+1}}\right)\|X\|_{r-1,(i+1) \gamma} .
\end{aligned}
$$

This shows (26). The proof is complete.

\section{Proof of Theorem 8}

As already announced, the proof of Theorem 8 is based on a combination of the antiadiabatic transform (Proposition 11) and the progressive diagonalization method (Corollary 14). Let us formulate it as a corollary.

Corollary 16. Let $r>2, i \geq 1, \gamma \in] 0, \frac{1}{2}[$, and $H$ be a self-adjoint operator with a pure point spectrum and the spectral decomposition $H=\sum_{n} E_{n} P_{n}$. Assume that the eigenvalues $\left\{E_{n}\right\}_{n=1}^{\infty}$ are ordered increasingly and satisfy (11). Further assume that $Y \in \mathcal{Y}(\infty, \gamma)$ is Hermitian and commutes with $H$, and $Z(t) \in \mathcal{Y}(r, i \gamma)$ is Hermitian, T-periodic and $C^{1}$ in the strong sense. If

$$
\|Y\|_{\infty, \gamma}+\|Z\|_{r, i \gamma} \leq \frac{c_{H}}{4 \pi C_{r+1}}
$$

then there exists a family $\mathcal{U}(t)$ of unitary operators on $\mathscr{H}$ which is $T$-periodic and $C^{1}$ in the strong sense and such that

$$
\mathcal{U}(t)(D+H+Y+Z(t)) \mathcal{U}(t)^{*}=D+H+A+B(t)
$$

where $A \in \mathcal{Y}(\infty, \gamma)$ is Hermitian, commutes with $H$ and fulfills

$$
\|A\|_{\infty, \gamma} \leq 2\left(\|Y\|_{\infty, \gamma}+\|Z\|_{r, i \gamma}\right)
$$

and $B(t) \in \mathcal{Y}(r-1,(i+1) \gamma)$ is T-periodic, Hermitian, continuously differentiable in the strong sense and satisfies

$$
\begin{aligned}
\|B\|_{r-1,(i+1) \gamma} \leq & \frac{1}{2 C_{r}} \exp \left(2 \frac{C_{r}}{C_{r+1}}\right) \\
& \times\left(\exp \left(4 C_{r} T\|Z\|_{r, i \gamma}\right)-1\right)\left(C_{H}+4\|Y\|_{\infty, \gamma}+2 C_{r}\|Z\|_{r, i \gamma}\right) .
\end{aligned}
$$

To prove Corollary 16 it suffices to set $\mathcal{U}(t)=U \exp (\mathrm{i} F(t))$ where $F(t)$ comes from Proposition 11 and $U$ comes from Corollary 14. Apart of this one applies the following elementary estimate: if the norm $\|X\|_{p, \delta}$ of a $T$-periodic family $X(t)$ formed by bounded operators is finite for some $p>1$ and $\delta \geq 0$ then the time average $\bar{X}$ of $X(t)$ over the period $T$ fulfills $\|\bar{X}\|_{p, \delta} \leq\|X\|_{p, \delta}$.

Equipped with Corollary 16 we are ready to approach the proof of Theorem 8 . 
Proof of Theorem 8 One starts from the Floquet Hamiltonian $K=D+H+V(t)$ and applies to it $q$ times Corollary 16, with the steps being enumerated by $i=1,2, \ldots, q$. In the $i$ th step one assumes that a strongly continuous function $J_{i-1}(t)$ with values in unitary operators on $\mathscr{H}$ has been already constructed so that

$$
K=J_{i-1}(t)\left(D+H+A_{i-1}+B_{i-1}(t)\right) J_{i-1}(t)^{*}
$$

with $A_{i-1} \in \mathcal{Y}(\infty, \gamma)$ being Hermitian and commuting with $H$, and $B_{i-1}(t) \in$ $\mathcal{Y}(p-i+1, i \gamma)$ being symmetric, $T$-periodic and $C^{1}$ in the strong sense. In the first step one sets $A_{0}:=0, B_{0}(t):=V(t)$ and $J_{0}(t):=1$.

Corollary 16 can be applied to the Floquet Hamiltonian $K_{i-1}:=D+H+A_{i-1}+B_{i-1}(t)$, with $r=p-i+1$, provided there is satisfied the assumption

$$
\left\|A_{i-1}\right\|_{\infty, \gamma}+\left\|B_{i-1}\right\|_{p-i+1, i \gamma} \leq \frac{c_{H}}{4 \pi C_{p-i+2}}
$$

Recall that the constant $C_{p}$ is given by (8). Under this assumption, there exists a strongly differentiable family of unitary operators $\mathcal{U}_{i}(t)$ such that

$$
K_{i}:=D+H+A_{i}+B_{i}(t)=\mathcal{U}_{i}(t) K_{i-1} \mathcal{U}_{i}(t)^{*}
$$

where $A_{i} \in \mathcal{Y}(\infty, \gamma)$ is symmetric and diagonal, and $B_{i}(t) \in \mathcal{Y}(p-i,(i+1) \gamma)$ is $T$ periodic, symmetric and strongly $C^{1}$. Moreover,

$$
\left\|A_{i}\right\|_{\infty, \gamma} \leq 2\left(\left\|A_{i-1}\right\|_{\infty, \gamma}+\left\|B_{i-1}\right\|_{p-i+1, i \gamma}\right)
$$

and

$$
\begin{aligned}
\left\|B_{i}\right\|_{p-i,(i+1) \gamma} \leq & \frac{1}{2 C_{p-i+1}} \exp \left(2 \frac{C_{p-i+1}}{C_{p-i+2}}\right)\left(\exp \left(4 C_{p-i+1} T\left\|B_{i-1}\right\|_{p-i+1, i \gamma}\right)-1\right) \\
& \times\left(C_{H}+4\left\|A_{i-1}\right\|_{\infty, \gamma}+2 C_{p-i+1}\left\|B_{i-1}\right\|_{p-i+1, i \gamma}\right)
\end{aligned}
$$

Finally, $J_{i}(t):=J_{i-1}(t) \mathcal{U}_{i}(t)^{*}$ is a family of unitary operators which is continuously differentiable in the strong sense and such that

$$
K=J_{i}(t)\left(D+H+A_{i}+B_{i}(t)\right) J_{i}(t)^{*} .
$$

To finish the proof we have to choose $\varepsilon>0$ sufficiently small so that if $\|V\|_{p, \gamma}<\varepsilon$ then condition (45) is satisfied in each step $i=1,2, \ldots, q$.

From (46) one derives by induction

$$
\left\|A_{i}\right\|_{\infty, \gamma} \leq \sum_{j=0}^{i-1} 2^{i-j}\left\|B_{j}\right\|_{p-j,(j+1) \gamma} .
$$

From here we deduce that inequalities (45) are satisfied for $i=1,2, \ldots, k$, provided the inequalities

$$
\sum_{j=0}^{i-1} 2^{i-1-j}\left\|B_{j}\right\|_{p-j,(j+1) \gamma} \leq \frac{c_{H}}{4 \pi C_{p-i+2}}
$$


are satisfied for the same range of indices. Furthermore, relations (45) and (47) imply that

$$
\left\|B_{i}\right\|_{p-i,(i+1) \gamma} \leq \phi_{i}\left(\left\|B_{i-1}\right\|_{p-i+1, i \gamma}\right)
$$

where

$$
\phi_{i}(y):=\frac{\exp \left(2 \frac{C_{p-i+1}}{C_{p-i+2}}\right)}{2 C_{p-i+1}}\left(\exp \left(4 C_{p-i+1} T y\right)-1\right)\left(C_{H}+\frac{c_{H}}{\pi C_{p-i+2}}+\left(2 C_{p-i+1}-4\right) y\right) .
$$

Set

$$
F_{i}(y):=2^{i-1} y+\sum_{j=1}^{i-1} 2^{i-1-j} \phi_{j} \circ \phi_{j-1} \circ \cdots \circ \phi_{1}(y), \quad i=1,2, \ldots, q .
$$

It follows from (49) that inequalities (48) are satisfied for $i=1,2, \ldots, k$, if it holds

$$
F_{i}\left(\left\|B_{0}\right\|_{p, \gamma}\right) \leq \frac{c_{H}}{4 \pi C_{p-i+2}}
$$

for the same range of indices.

Recall that $B_{0}(t)=V(t)$. From this discussion it is clear that condition (45) is satisfied in all steps $i=1,2, \ldots, q$, provided $\|V\|_{p, \gamma} \leq \varepsilon$ and $\varepsilon>0$ is chosen so that

$$
\forall i \in\{1,2, \ldots, q\}, \forall y \in[0, \varepsilon], F_{i}(y) \leq \frac{c_{H}}{4 \pi C_{p-i+2}} .
$$

But all functions $\phi_{i}(y)$ are continuous, strictly increasing and satisfy $\phi_{i}(0)=0$. Consequently, the same is true for all functions $F_{i}(y)$. Hence the following choice of $\varepsilon$ will do:

$$
\varepsilon=\min \left\{F_{i}^{-1}\left(\frac{c_{H}}{4 \pi C_{p-i+2}}\right) ; 1 \leq i \leq q\right\} \text {. }
$$

This completes the proof of Theorem 8 .

\section{Acknowledgments}

The authors wish to acknowledge gratefully partial support from the following grants: grant No. 201/05/0857 of the Grant Agency of the Czech Republic (P. Š.), grant No. MSM 6840770010 of the Ministry of Education of the Czech Republic (O. L.), and grant No. LC06002 of the Ministry of Education of the Czech Republic (O. L. and P. S.).

\section{References}

[1] Asch J., Duclos P., Exner P., Stability of driven systems with growing gaps, quantum rings, and Wannier ladders, J. Stat. Phys. 92 (1998) 1053-1070.

[2] Astaburuaga M. A., Bourget O., Corts V. H., Fernndez C., Floquet operators without singular continuous spectrum J. Funct. Anal. 238 (2006) 489-517. 
[3] Barbaroux J. M., Joye A., Expectation values of observables in time-dependent quantum mechanics, J. Stat. Phys. 90 (1998) 1225-1249.

[4] Bunimovich L., Jauslin H. R., Lebowitz J. L., Pellegrinotti A., Nielaba P., Diffusive energy growth in classical and quantum driven oscillators, J. Stat. Phys. 62 (1991) 793-817.

[5] De Bièvre S., Forni G., Transport properties of kicked and quasiperiodic Hamiltonians, J. Stat. Phys. 90 (1998) 1201-1223.

[6] Bhatia R., Rosenthal P., How and why to solve the operator equation $A X-X B=Y$, Bull. London Math. Soc. 29 (1997) 1-21.

[7] Bourget O., Singular continuous Floquet operators for systems with increasing gaps, J. Math. Anal. Appl. 276 (2002) 28-39.

[8] Bourget O., Singular continuous Floquet operator for periodic quantum systems, J. Math. Anal. Appl. 301 (2005) 65-83.

[9] Combes J.-M., Connection between quantum dynamics and spectral properties of time-evolution operators, in "Differential Equations and Applications to Mathematical physics", W. F. Ames, E. M. Harrell and J. V. Herod eds. (Academic Press, Boston, 1993) pp. 59-68.

[10] Combescure M., The quantum stability problem for time-periodic perturbations of the harmonic oscillator, Ann. Inst. Henri Poincaré 47 (1987) 62-82, Erratum: Ann. Inst. Henri Poincaré 47 (1987) 451-454.

[11] Combescure M., Spectral properties of a periodically kicked quantum Hamiltonian, J. Stat. Phys. 59 (1990) 679-690.

[12] Combescure M., Recurrent versus diffusive dynamics for a kicked quantum oscillator, Ann. Inst. H. Poincaré 57 (1992) 67-87.

[13] Duclos P., Lev O., Šťovíček P., Vittot M.: Progressive diagonalization and applications, in "Operator Algebras and Mathematical Physics", J.-M. Combes et al. eds. (The Theta Foundation, Bucharest, 2003) pp. 75-88.

[14] Duclos P., Soccorsi E., Šťovíček P., Vittot M.: Dynamical localization in periodically driven quantum systems, in "Advances in Operator Algebras and Mathematical Physics", F.-P. Boca, O. Bratteli, R. Longo and H. Siedentop eds. (The Theta Foundation, Bucharest, 2005) pp. 57-66.

[15] Enss V., Veselić K., Bound states and propagating states for time-dependent Hamiltonians, Ann. Inst. H. Poincaré 39 (1983) 159-191.

[16] Guarneri I., Mantica G., On the asymptotic properties of quantum dynamics in the presence of a fractal spectrum, Ann. Inst. H. Poincaré A 61 (1994) 369-379. 
[17] Hagedorn G. A., Loss M., Slawny J., Non-stochasticity of time-dependent quadratic Hamiltonians and the spectra of canonical transformations, J. Phys. A: Math. Gen. 19 (1986) 521-531.

[18] Howland J. S., Floquet operators with singular spectrum, I, Ann. Inst. H. Poincaré, 50 (1989) 309-323.

[19] Howland J. S., Floquet operators with singular spectrum, II, Ann. Inst. H. Poincaré, 50 (1989) 325-334.

[20] Howland J. S., Floquet operators with singular spectrum, III, Ann. Inst. H. Poincaré, 69 (1998) 265-273.

[21] Jauslin H., Lebowitz J. L., Spectral and stability aspects of quantum chaos, Chaos, 1 (1991) 114-121.

[22] Joye A., Absence of absolutely continuous spectrum of Floquet operators, J. Stat. Phys. 75 (1994) 929-952.

[23] Joye A., Upper bounds for the energy expectation in the time-dependent quantum mechanics, J. Stat. Phys. 85 (1996) 575-606.

[24] Krein S. G., Linear Differential Equations in Banach Spaces (AMS, Providence, Rhode Island, 1971)

[25] McCaw J., McKellar B., On the continuous spectral component of the Floquet operator for a periodically kicked quantum system J. Math. Phys. 46 (2005) 103503.

[26] Nenciu G., Floquet operators without absolutely continuous spectrum, Ann. Inst. H. Poincaré A 59 (1993) 91-97.

[27] Nenciu G., Adiabatic theory: Stability of systems with increasing gaps, Ann. Inst. H. Poincaré A 67 (1997) 411-424.

[28] de Oliveira C. R., Some remarks concerning stability for nonstationary quantum systems, J. Stat. Phys. 78 (1995) 1055-1065.

[29] de Oliveira C. R., Simsen M. S., A Floquet operator with pure point spectrum and energy instability, Ann. Inst. H. Poincaré, to appear.

[30] Reed M., Simon B., Methods of Modern Mathematical Physics III, Academic Press, San Diego, 1979. 